\title{
Day Care Laproscopic Cholecystectomy - A Prospective Study
}

\author{
Ahi Kuldip Singh ${ }^{1}$,Sood Dinkar ${ }^{1}$,Jain Aditya ${ }^{2}$, Kumar Avnish $^{2}$, \\ Sood Singla Heena ${ }^{3}$ \\ ${ }^{I}$ Department Of Surgery, Government Medical College, Patiala, India. \\ ${ }^{2}$ Department Of Physiology, Government Medical College, Patiala, India \\ ${ }^{3}$ Department Of Pathology, Government Medical College, Patiala, India
}

\begin{abstract}
A study was conducted at Rajindra Hospital Patiala in fifty cases undergoing Laparoscopic cholecystectomy from August 2010 to August 2011. The study was conducted on selected patients who fulfilled the pre-set inclusion criteria. The patients were discharged on the same day of surgery as done in Day Care Surgery. The feasibility and outcome of Day care Laparoscopic Cholecystectomy was studied. Patients who presented with the diagnosis of cholelithiasis and gave consent for Day Care Laparoscopic Cholecystectomy were included in the study. Selection criteria included age less than sixty years, ASA grade I and II, no signs and symptoms of acute cholecystitis, normal liver function tests. Early ambulation after surgery, complications, patient requiring overnight stay, patients requiring readmission and other factors were studied. Of the total 50 patients who underwent laparoscopic cholecystectomy, 45 were discharged from the hospital on same day of the surgery. 5 patients were not discharged due to either nausea/vomiting or severe abdominal pain. 2 out of 50 patients required readmission to the hospital but were managed conservatively.
\end{abstract}

\section{Introduction}

Laparoscopic cholecystectomy has replaced open cholecystectomy for the treatment of gall stone disease and now treatment of choice for symptomatic cholelithiasis ${ }^{[1]}$.Cholelithiasis was first described in 1420 by a Florentine pathologist,Antonio Benivenius ${ }^{[2],[3] .}$ Further, Jean-Louis Petit, the founder of gall bladder surgery in 1733 suggested removal of gall stones and drainage of gall bladder ${ }^{[4]}$. The operative treatment of gall stones really began with the pioneer work of Ephraim Mcdowell (1780-1830), who in 1809, was the surgeon to open the abdomen with the object of removing an ovarian cyst ${ }^{[5]}$.

Cholecystectomy is the commonest operation of the biliary tract and the second most common operative procedure performed today ${ }^{[6],[7]}$. The first open cholecystectomy was performed on July $15^{\text {th }}, 1882$, by a German surgeon Carl Johann August Langenbuch at the Lazarus Krankenhaus, Berlin, on a 43 year old $\operatorname{man}^{[8],[9]}$. With the advances in the technique during the later years, the Russian Lukichev in 1983 performed the first laparoscopic cholecystectomy, but its publication was limited to the Russian literature, and it remained unknown ${ }^{[8],[10]}$. However, the first actual laparoscopic cholecystectomy was performed by Professor Dr Erich Muhe in Boblingen, Germany, on September 12, 1985. he performed 94 such procedures before Phillip Mouret had performed his first laparoscopic cholecystectomy in $1987^{[11],[13]}$. However, this innovative technique was not widely accepted. Instead, it was Mouret who was responsible for laparoscopic surgery achieving medical respectability by performing the first laparoscopic cholecystectomy ${ }^{[8,14]}$. In spite of his early rejection in 1986, at the $109^{\text {th }}$ German Surgical Society(GSS) Congress on April , 1992 , Muhe received the GSS anniversary Award for his pioneering work in endoscopic surgery ${ }^{[13]}$.

In India, Professor Tehempton E Udwadia ${ }^{[15]}$ from Mumbai performed the first laparoscopic cholecystectomy in 1990 and presented the paper at the $10^{\text {th }}$ world congress of digestive surgery at New Delhi in the same year (1990)

Since then laparoscopic cholecystectomy has received an immense amount of publicity as the revolutionary new method for the treatment of gall stone disease ${ }^{[16]}$. Initially there was hesitation on the part of the surgeons to accept this laparoscopic procedure but the advantages were quiet evident and in addition there was rising patient demand for the procedure, so now over the years laparoscopic cholecysytectomy has emerged over the open cholecystectomy as the "Gold Standard" for surgical treatment of symptomatic gall stones ${ }^{[8]}$. Rapidly escalating patient demand and the initial limited availability of instrumentation and appropriately trained surgeons combined to create an aura of uniqueness for laparoscopic cholecystectomy. Although exciting, this technique represents not the revolution but rather the evolution of surgery and the culmination of nearly 100 years.

Minimal invasive surgery holds an important position in today's practice. A large number of surgical procedures are performed laparoscopically with laparoscopic cholecystectomy being one of the most popular. The benefits of laparoscopic cholecystectomy compared with open surgery are well known ${ }^{[17]}$. 
Laparoscopic operative procedures have revolutionized surgery with many advantages: a smaller and a more cosmetic incision, reduced blood loss, reduced postoperative stay and pain, decreased risk of surgery and anesthesia related complications, which is reflected in patient's earlier return to normal routine life and work activities $^{[18]}$

The new procedure has been widely accepted and adopted by surgical community and has become new "gold standard" for management of cholelithiasis ${ }^{[15]}$. However as the experience advances further, a few centers have recently shown that the operation is safe and feasible even as an out patient procedure in properly selected and pre counseled patients ${ }^{[19]}$.

So one of the latest advancement in the field of laparoscopic surgery is the introduction of Day Care surgery. Day care surgery is described as the admission of selected patients for a planned surgical procedure, returning home on the very same day after the operative procedure ${ }^{[20]}$. Ability to provide high quality and cost effective care has made ambulatory surgery one of the fastest growing area in the health care system all over the world. Primary aim of the day care surgery is to avoid special hospitalization but the patient safety is the ultimate goal of the concept ${ }^{[21]}$. Rapid recovery after laparoscopic cholecystectomy and increasing experience with its post operative course has led to progressively shorter post operative period and the recent trend of day care surgery without an overnight stay at hospital. The advantages of patient satisfaction and cost effectiveness were highly attractive to surgeons and hospital administrators ${ }^{[22,23]}$.

Although in some countries like U.S.A. and Canada the concept of day care laparoscopic cholecystectomy has already been widely accepted with recent reports focusing less on feasibility but rather on the possibility to apply wider inclusion criteria. In European countries the concept of treatment of symptomatic cholelithiasis on an outpatient basis is still infrequent ${ }^{[24]}$. Reddick and Oslen first reported an ambulatory laparoscopic cholecystectomy in $1990^{[25]}$. The safety of ambulatory laparoscopic cholecystectomy has been reported through its increased use $\mathrm{e}^{[22,26]}$.

Benefits of Day Care Surgery

1) Reduced hospital stay.

2) Early resumption of day to day activity.

3) Cost effectiveness.

4) Reduces anxiety of the patient.

5) Faster recovery in familiar surroundings.

6) Improved patient satisfaction.

7) Decreased hospital acquired infections.

The feasibility of Day Care laparoscopic cholecystectomy is very much dependent on the post operative period of the patient. An uneventful post operative period is quite essential for the patient to gain confidence in the concept. Reports over the last few years of treating patients with symptomatic cholelithiasis as an outpatient setting have indicated a significant reduction in costs without increasing the morbidity and mortality. The main emphasis in day care laparoscopic cholecystectomy surgery is on pain management and relief from nausea and vomiting. After any surgery rapid recuperation of patient's autonomy, shortened hospital stay, decreased morbidity and costs are directly related to the post operative pain management. Post operative pain is unpredictable, which explains the need for adequate prevention of pain before the patient wakes up from anesthesia ${ }^{[27]}$. Thus pain relief and patient comfort during the early post operative period becomes increasingly important, as the need for analgesics may delay discharge. Causes of pain can be distension induced neuropraxia of phrenic nerves, residual intra abdominal gas, wound size, trauma to parietal peritoneum, presence of drains, anesthetic drugs and their post operative effect, socio cultural and individual factors. To decrease post operative pain use of injection bupivacaine has been used at the port sites. This has reportedly decreased the post operative pain.

The safety of Day Care laparoscopic cholecystectomy has been demonstrated through its increased use $(22,26)$. However, practice of its use in public sector hospital setting is still uncommon. Even in a country like India were the surgical advancement is moving at a very fast pace, the concept of Day Care surgery is still not widely acceptable to the practicing surgeons. Most of the surgeons in India follow the concept of observing the patient overnight post laparoscopic chloecystectomy. Though some cases of Day Care surgery have been reported in the literature from India.

The concept of Day Care surgery is very beneficial for a developing country because it not only leads to cost containment but also better utilization of the health care resources The purpose of present study is to evaluate feasibility and outcome of day care laparoscopic cholecystectomy in a public sector hospital (Rajindra Hospital Patiala, Punjab, India). Where 50 patients selected according to a pre-set inclusion criteria were subjected to Day Care laparoscopic cholecystectomy. 


\section{Materials And Method}

The study was conducted with 50 patients undergoing laparoscopic cholecystectomy at Rajindra Hospital Patiala, Punjab, India. The patients presented to the surgical outpatient department with confirmed diagnosis of gall bladder stones but not having any symptoms of acute cholecystitis were considered. Patients who gave informed consent after full explanation of day care process were electively admitted for an ambulatory laparoscopic cholecystectomy. Patients data was collected on a pre designed computer based Performa consisting of various data like demography, disease, treatment, outcome and follow up.

\section{Selection criteria}

Inclusion criteria

1. Signs and symptoms suggestive of symptomatic gall bladder stones.

2. Absence of clinical and radiological findings of acute cholecystitis, biliary pancreatitis or empyma gall bladder at time of admission.

3. American society of Anesthesiologists grade I and II ASA grade I healthy patient. no medical problems

ASA grade II mild systemic illness

4. Patients under 60 years of age.

5. Normal liver function tests.

6. Body weight less than $100 \mathrm{~kg}$.

\section{Exclusion criteria}

1. Multiple co morbities (severe hypertension, uncontrolled diabetes, tuberculosis, immunocompromised patients).

2. Coagulation disorders.

3. Adverse anesthetic history.

4. Other procedures performed along with cholecystectomy or undergoing bile duct exploration, per operative finding cholecystitis or suspect/proven malignancy.

5. American society of anesthesiologists grade III, IV, V

ASA grade III severe systemic disease, but not incapacitating

ASA grade IV severe systemic disease that is constant threat to life

ASA grade $\mathrm{V}$ moribund, not expected to survive without operation.

6. Unavailability of competent adult to accompany the patient will be excluded from the study.

\section{Anesthesia protocol}

All patients were sent to the general anesthesia clinic for pre anesthesia evaluation. After attaining anesthetic fitness for surgery .Patients were posted for early morning surgery, patients were kept fasting over night. Tablet Alprazolam (.25mg) was given bed time and at 6.00 am on the day of surgery with a sip of water. General anesthesia established using following protocol

\section{Pre medication}

Injection Phenargan $(25 \mathrm{mg})$ i.m.

Injection Midazolam(2 mg)i.m.

Given 30 minutes before surgery.

\section{Induction And Intubation}

I.v. line secured. Pre-Oxygenation done for 5 minutes with $100 \%$ oxygen $\left(\mathrm{o}_{2}\right)$. Injection Ramosetron $(0.3 \mathrm{mg})$ i.v. given. Induction done with Injection Glycopyrrolate $(0.1 \mathrm{mg})$ i.v, injection Fentanyl $(2$ micro $\mathrm{gm} / \mathrm{kg}$ ) i.v., Injection Propofol (1-1.5 mg/kg).i.v, relaxation attained with Injection Atracurium $(0.3-0.5 \mathrm{mg} / \mathrm{kg})$ i.v. All the patients were given parenteral prophylactic antibiotic.

\section{Maintenance}

Maintenance was done with Oxygen, N2O, Isoflurane, Atracurium and Fentanyl was given when required. Regular monitoring of haemodynamic parameters including pulse rate, blood pressure, oxygen saturation and electro cardiogram was done during surgery. At the end of surgery reversal with Myopyrrolate (Glycopyrrolate + Neostigmine) was given. Suction and extubation was done and the patient was shifted to recovery when fully conscious and co-operative. Post operatively patient was monitored for vitals and immediate complications. Oral liquids were allowed 6 hours after surgery. 


\section{Operative technique}

Operation was scheduled in the early morning session of the operating list .Patient were kept fasting overnight. The operative field prepared and draped. All patients recieved a dose of pre operative prophylactic antibiotic. Four port technique for laparoscopic cholecystectomy was used. Two $10 \mathrm{~mm}$ ports and two $5 \mathrm{~mm}$ ports were used. $10 \mathrm{~mm}$ ports in the umblical and epigastric region. $5 \mathrm{~mm}$ ports in the right hypochondrium and anterior axillary line (subcostal). Pneumoperitoneum was created by inserting Veeres needle in the infraumblical region. Once the pneumoperitoneum was created a $10 \mathrm{~mm}$ port was introduced and a telescope was put in. After the abdominal survey, rest of the ports were put under direct vision i.e. the $10 \mathrm{~mm}$ port in the epigastric region, $5 \mathrm{~mm}$ port in the right hypochondrium and another $5 \mathrm{~mm}$ port in the anterior axillary line(subcostal). The patient was placed in reverse trendelburg's(fowler's)position with the patient head up and tilted to the left and the surgeon standing on the left side of the patient. Gall bladder was grasped from the fundus through a $5 \mathrm{~mm}$ port and retracted. Dissection was done at the level of calot's triangle. Cystic artery and duct were defined. Clipping of the cystic duct and cystic artery was done separately using liga clips. Gall bladder was removed from a 10 $\mathrm{mm}$ port (epigastric). Abdominal cavity was washed with normal saline to remove all the clots and spilled biliary content if any. Complete haemostasis was achieved. All port sites were injected with $0.5 \%$ bupivacaine before closure.

\section{Post operative course}

Patients were given antiemetic (ramosetron) i.v nsaids(lornaxicam) i.v in the recovery room itself. Assessment of the post operative symptoms like nausea, vomiting, post operative pain was done in the recovery room itself. Patients were allowed to take a liquid diet 6 hours after surgery with a repeat dose of both anti emetic and pain killer injection. Patients who accepted orally and did not develop any post operative symptoms/complication were discharged on the very same day of the surgery. These patients were instructed to report back to the emergency if any complications appear. The discharged patients were followed up in surgical outpatient department on $2^{\text {nd }}, 5^{\text {th }}$ and $10^{\text {th }}$ post operative day. But the patients who developed any post operative fever, persistent vomiting, severe pain not relieved by medication were not discharged from the hospital and were taken care of under the supervision of the surgical team at the hospital itself.

\section{Criteria For Discharge}

1. Stable vital signs for $>30$ minutes.

2. No new signs or symptoms after the surgery.

3. No active bleeding or oozing.

4. Able to walk without support.

5. Accepting orally with no vomiting.

6. No or minimal pain controllable with oral analgesics.

7. Passed urine post surgery.

8. Responsible person is present at home to take care of the patient.

9. No surgical complications.

\section{Instructions On Discharge}

1. Written instructions regarding the post operative medication of the patient.

2. Verbal instructions regarding the oral intake, complications, adequate rest.

3. Contact no of the concerned resident doctor.

4. Instructions on how to look for complications and its management and if not relieved report to hospital immediately.

\section{Contraindications To Day Care Surgery}

1. Mentally retarded patient or psychologically unstable

2. Having highly infectious disease

3. Poor patient acceptance

4. No facility for post operative care at the home of the patient

5. No availability of nursing care near the home of patient in case of any complication

6. Patients who required extensive post operative monitoring

7. Long distance from home i.e. more than 40 kilometers away from the hospital. 


\section{Observations and Results}

A total of fifty $(n=50)$ patients were selected for day care laparoscopic cholecystectomy according to a preset selection criteria .

1) Signs and symptoms suggestive of symptomatic cholelithiasis.

2) Absence of clinical and radiological signs of acute cholecystitis.

3) American society of anesthesiologists grade I and II ASA grade I healthy patient, no medical problems

ASA grade II mild systemic illness.

4) Patients under 60 years of age.

5) Normal liver function tests.

6) Body weight less than $100 \mathrm{~kg}$.

The selected patients were between 21-59 years of age, majority (45 out of 50) were women. The patients presented to the outpatient department with symptomatic cholelithiasis. They were counseled and motivated for day care surgery. All the patients underwent routine investigations and a pre anesthetic check up for fitness for surgery. Patients with ASA grade I and II were selected for the study.

Patients demographic data and presenting symptoms are given in

Table no 1 Demographic data and presenting symptoms (TABLE NO 1)

\begin{tabular}{|l|l|}
\hline Demographic data $\mathbf{n}=\mathbf{5 0}$ & No. $(\%)$ \\
\hline Age & \\
\hline Mean \pm SD & $39.66 \pm 11.33$ \\
\hline Range & $21-59$ yrs \\
\hline Sex & \\
\hline Female & $45(90)$ \\
\hline Male & $5(10)$ \\
\hline & \\
\hline ASA & $35(70)$ \\
\hline ASA- I & $15(30)$ \\
\hline ASA- II & \\
\hline & \\
Presenting symptoms & \\
\hline Recurrent biliary pain & $45(90)$ \\
\hline Previous cholecystitis & $4(8)$ \\
\hline Previous biliary pancreatitis & $1(2)$ \\
\hline Admission & \\
\hline Elective & $50(100)$ \\
\hline
\end{tabular}

Operative procedure and peroperative findings are given in Table 2

\begin{tabular}{|l|l|}
\hline Perioperative data $\mathbf{n}=\mathbf{5 0}$ & No. $(\%)$ \\
\hline Operative procedure & \\
\hline Four port lap. Cholecystectomy & $50(100)$ \\
\hline Peroperative diagnosis & \\
Uncomplicated gall bladder & $45(90)$ \\
\hline Obscure/abnormal anatomy & 0 \\
\hline Small contracted gall bladder & $2(4)$ \\
\hline Mucocele & $3(6)$ \\
\hline Empyema & 0 \\
\hline Open cholecystectomy & 0 \\
\hline \multicolumn{2}{|c|}{} \\
Mean operative time(minutes) & $5 \pm \pm 0.74$ \\
\hline Mean hospital stay (hours) & \\
\hline Histopathology & $50(100)$ \\
\hline Chronic cholecystitis & 59.08 \\
\hline
\end{tabular}

All the selected patients underwent four port laparoscopic cholecystectomy. No conversion to open cholecystectomy was done in any case. Mean operative time for Laparoscopic cholecystectomy was $59 \pm 17.08$ SD minutes. Mean hospital stay after surgery, of the 45 patients who were discharged on the same day of the procedure was $9 \pm 0.74$ hours. Bile/stone spillage occurred in 8 out of 16 patients. For this the peritoneal cavity was thoroughly washed with normal saline until clear fluid was aspirated and spilled stones were removed. Cystic artery bleed was controlled by diathermy followed by clipping of the artery.Perioperative complications $(20 \%)$ were observed and are reported in table 3 as follows 
Table 3

\begin{tabular}{|l|l|}
\hline Perioperative complications $\mathbf{n}=\mathbf{5 0}$ & No. $(\%)$ \\
\hline Port site bleeding & 0 \\
\hline Bile/stone spillage & $8(16)$ \\
\hline Cystic artery bleeding & $2(4)$ \\
\hline Sub hepatic collection & 0 \\
\hline Other vascular injury & 0 \\
\hline CBD injury & 0 \\
\hline Bowel injury & 0 \\
\hline Pancreatitis & 0 \\
\hline
\end{tabular}

No statistically significant association was observed in patients having perioperative complications with co morbidities, ASA level, diagnosis at admission, ultrasound findings, white blood count, perioperative finding, and operative time.

The patients undergoing day care laparoscopic cholecystectomy were evaluated on three parameters i.e. pain, nausea and vomiting. They were further classified into no symptoms, mild, moderate and severe categories with patients falling in mild category requiring no medication, moderate category requiring oral medication and patients under severe category requiring intravenous medication.

Table no 4

\begin{tabular}{|l|c|c|l|c|c|}
\hline Pain & $\begin{array}{l}\text { Post } \\
\text { operative }\end{array}$ & At Discharge & $\begin{array}{l}2^{\text {nd }} \\
\text { follow up }\end{array}$ & $\begin{array}{l}5^{\text {th }} \\
\text { up }\end{array}$ & $\begin{array}{l}10^{\text {th }} \text { follow day } \\
\text { follow up }\end{array}$ \\
\hline No pain/ & $10(20 \%)$ & $25(50 \%)$ & $22(44 \%)$ & $44(88 \%)$ & $46(92 \%)$ \\
Mild & $18(36 \%)$ & $20(40 \%)$ & $24(48 \%)$ & $5(10 \%)$ & $4(8 \%)$ \\
\hline Moderate & $20(40 \%)$ & $3(6 \%)$ & $4(8 \%)$ & 0 & 0 \\
\hline Severe & $2(4 \%)$ & $2(4 \%)$ & 0 & $1(2 \%)$ & 0 \\
\hline
\end{tabular}

Table no 5

\begin{tabular}{|l|c|c|}
\hline Nausea & Post operative & $2^{\text {nd }}$ day follow up \\
\hline No symptoms/ & $5(10 \%)$ & $28(56 \%)$ \\
Mild (1) & $25(50 \%)$ & $18(36 \%)$ \\
\hline Moderate (2) & $18(36 \%)$ & $4(8 \%)$ \\
\hline Severe (3) & $2(4 \%)$ & 0 \\
\hline
\end{tabular}

Table no 6

\begin{tabular}{|l|c|c|}
\hline Vomiting & Post operative & $2^{\text {nd }}$ day follow up \\
\hline No symptoms/ & $41(82 \%)$ & $48(96 \%)$ \\
Mild (1) & $4(8 \%)$ & $1(2 \%)$ \\
\hline Moderate (2) & $3(6 \%)$ & $1(2 \%)$ \\
\hline Severe (3) & $2(4 \%)$ & 0 \\
\hline
\end{tabular}

Post operative data

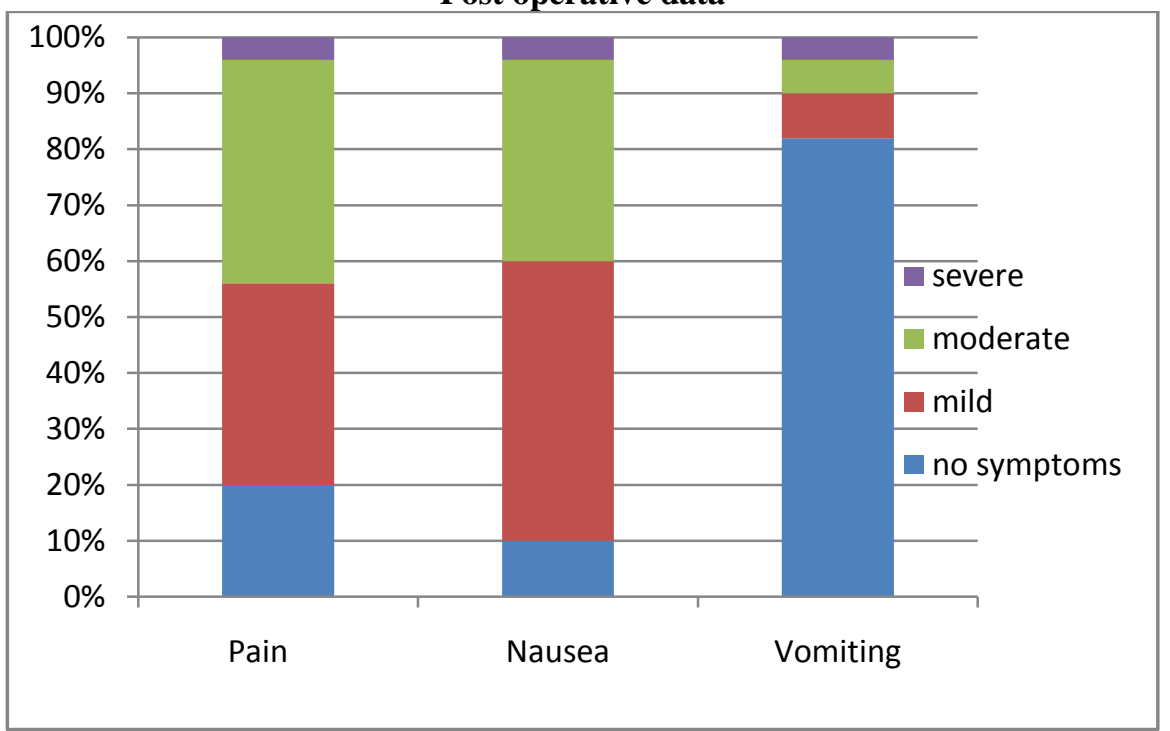

On $2^{\text {nd }}$ post operative day 


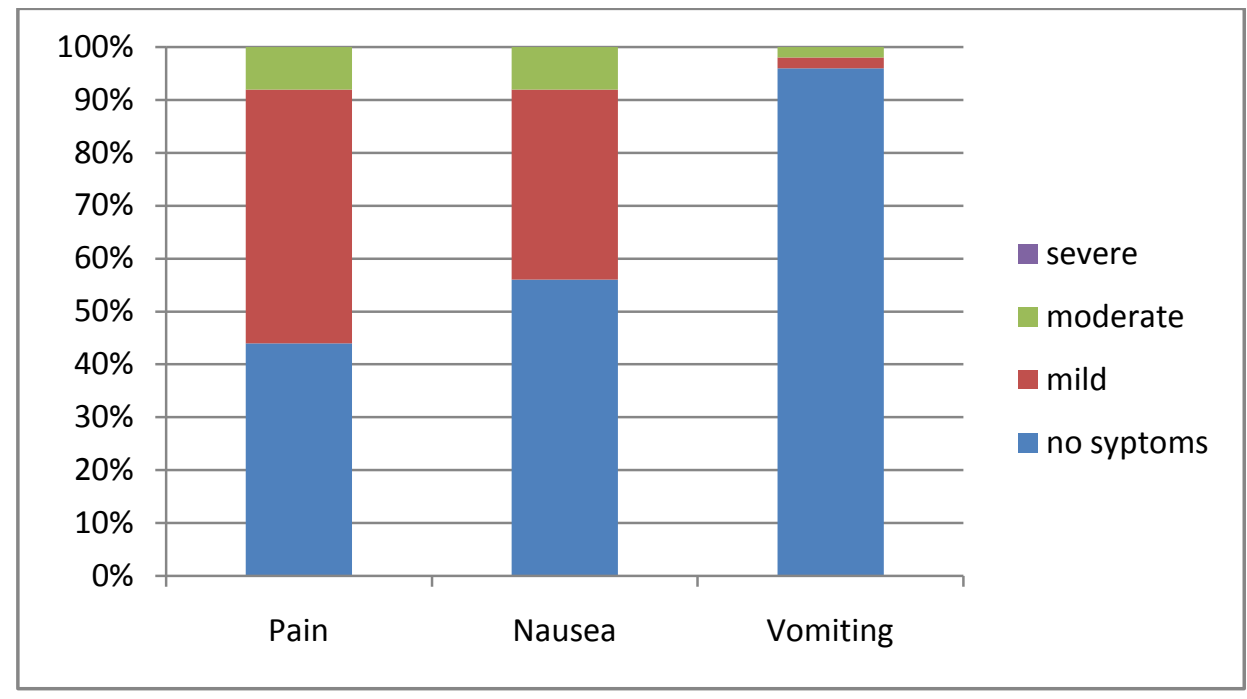

$5^{\text {th }}$ and $10^{\text {th }}$ day follow results of patients

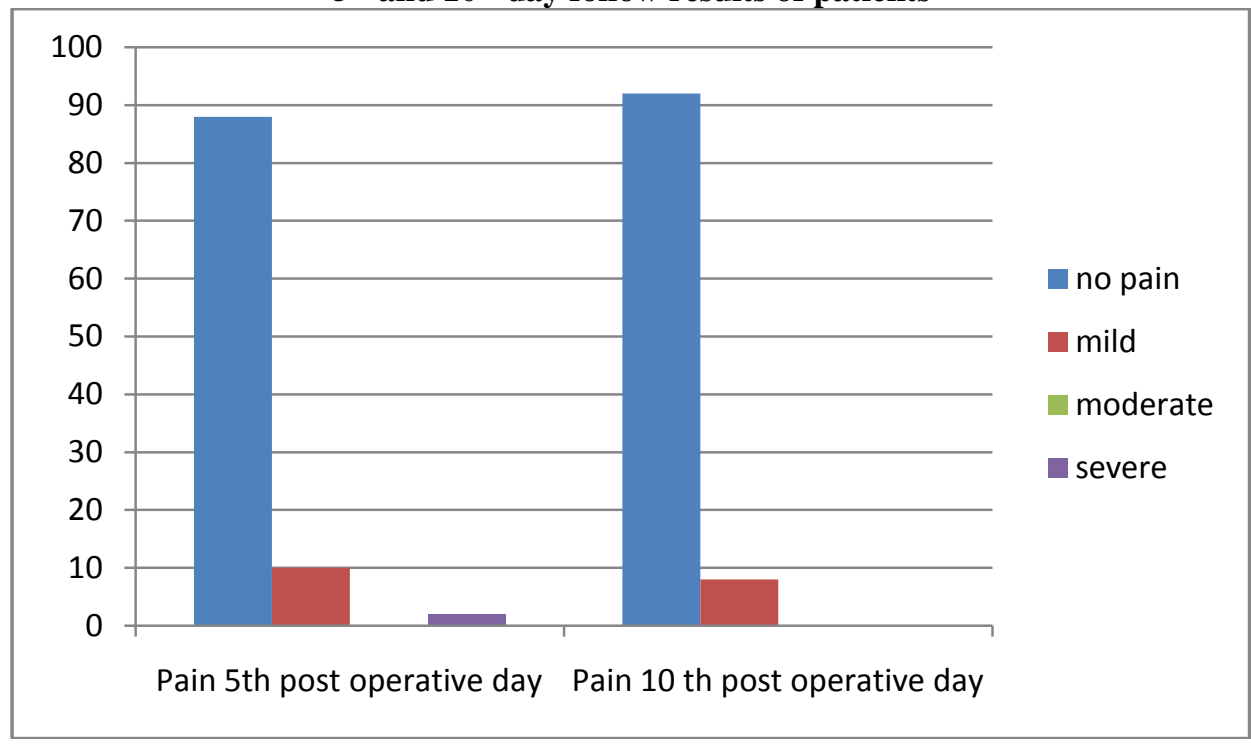

The patients who suffered from either moderate or severe pain were given oral lornoxicam and intravenous injection lornoxicam respectively. Similarly patients who developed moderate or severe post operative nausea/vomiting were given either oral or intravenous antiemetic. Forty five (90\%) patients were discharged successfully after 8-11 hours of observations in day care ward. Of the remaining five patients, two were admitted because of severe nausea/vomiting, two patients for excessive pain abdomen and one admitted because of patient's preference Unplanned admission and readmission rate was $10 \%$ and $4 \%$, respectively

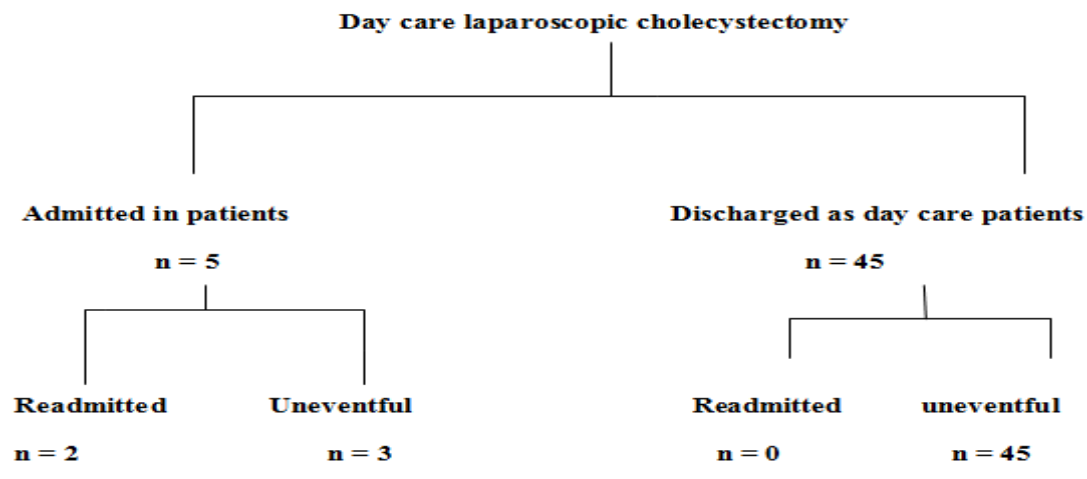


Table 7 Unplanned admission and readmission rate

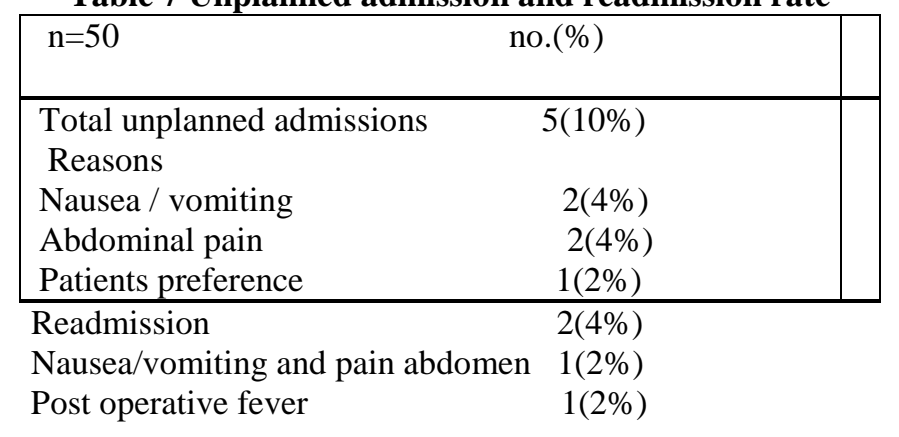

Out of the five patients who could not be discharged as Day care cases, two patients were readmitted to the hospital on the $4^{\text {th }}$ and $5^{\text {th }}$ post operative day respectively. One patient was readmitted because of postoperative fever on fourth postoperative day and the other patient had nausea / vomiting with pain abdomen and readmitted on the $5^{\text {th }}$ post operative day. Both the readmitted patients were subjected to routine blood tests, liver function tests and ultrasound abdomen. The blood tests of both the patients were found to be normal. Ultrasound abdomen showed no abdominal collection. Both the patients were managed conservatively and discharged in a stable condition.
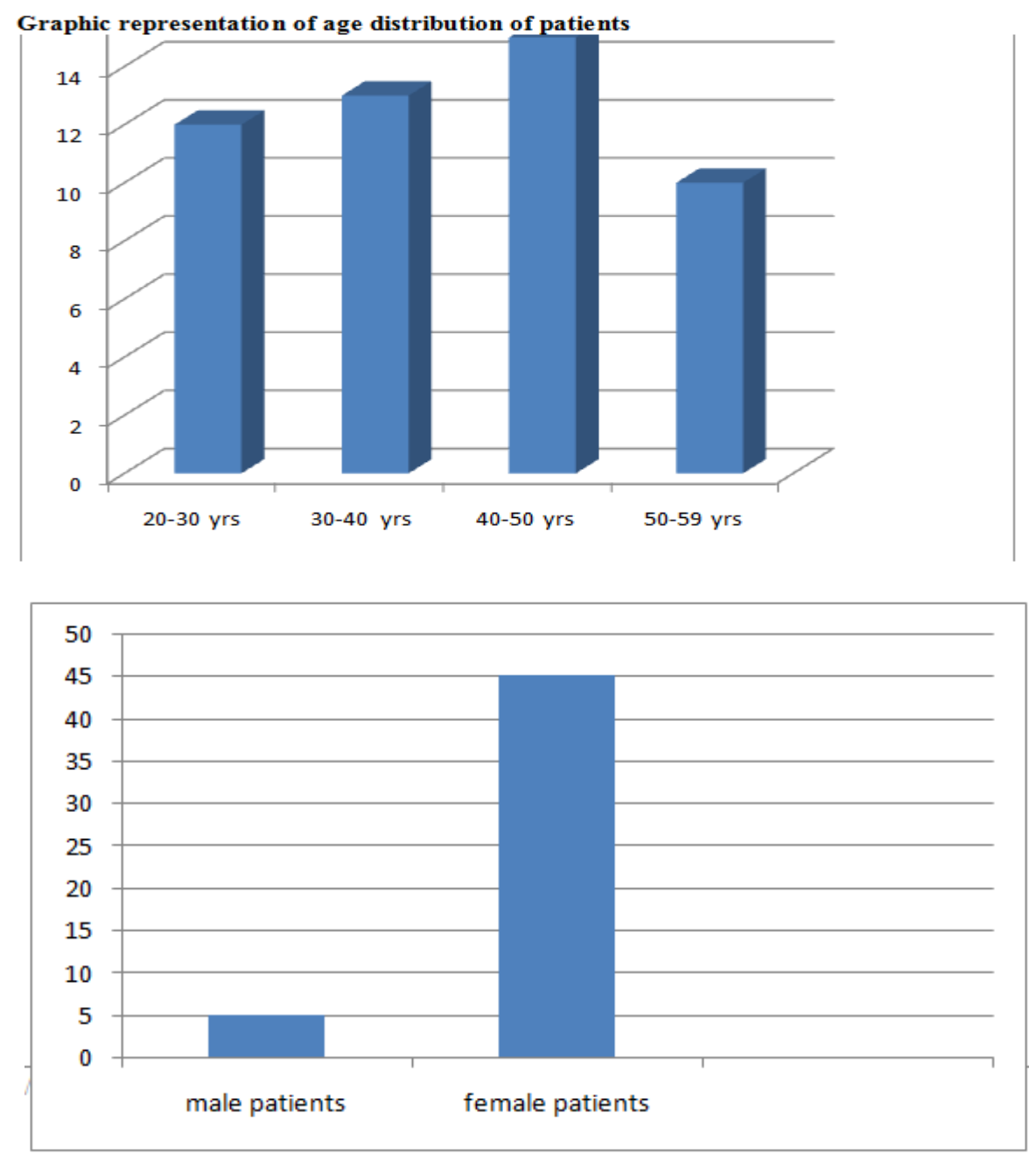

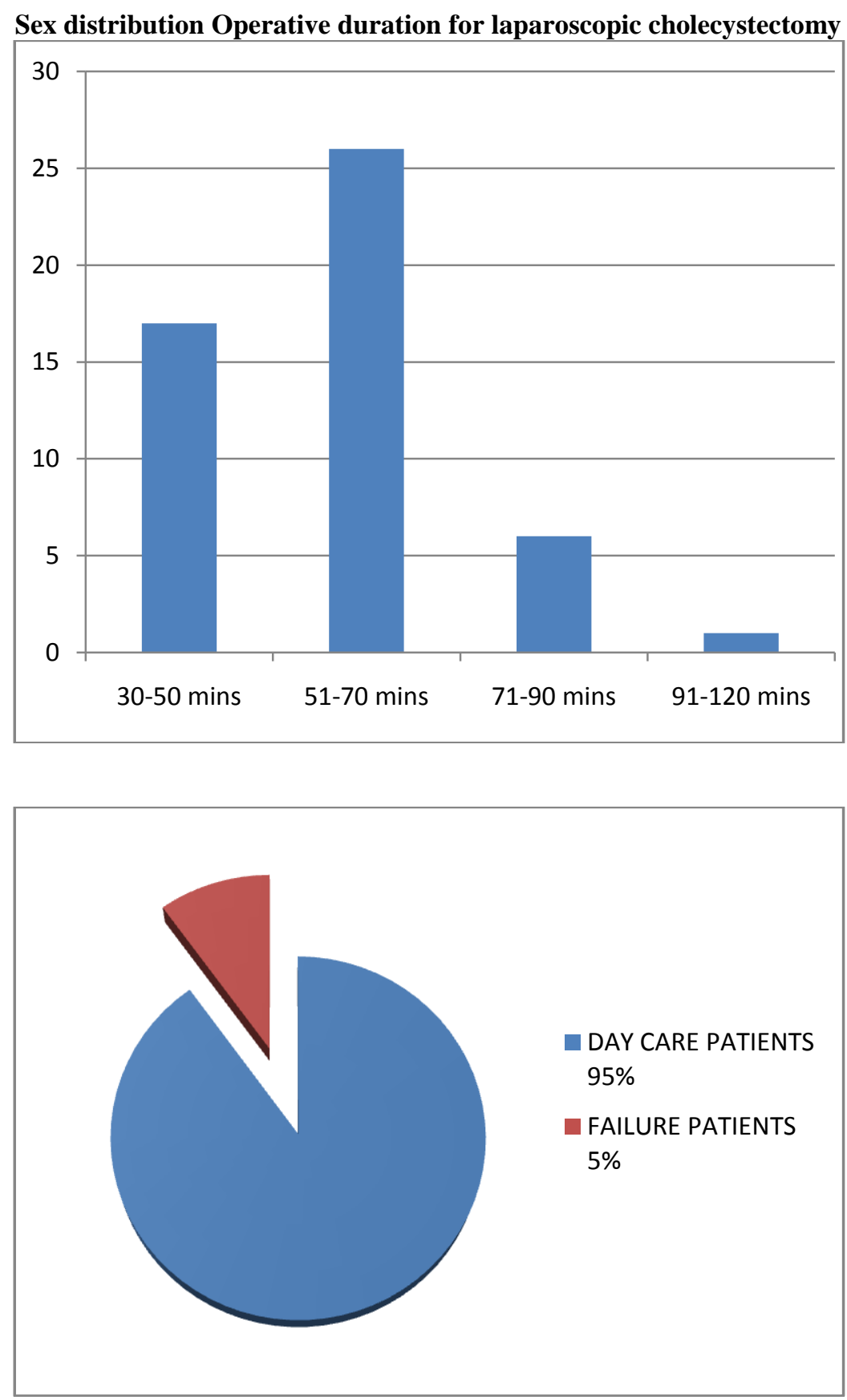

\section{Discussion}

With growing economic pressure, some centers have advocated routine application of Day Care laparoscopic cholecystectomy in all patients with no pre selection criteria. However day care surgery involves a shift of postoperative care from the hospital to the home of the patient after discharge. Recruitment of high risk patients presents a challenge to the limits of safe practice, particularly during the early postoperative day. Selection of the patients for day care surgery has been given utmost importance in every study conducted either in India or abroad. Though there are no set pattern of guidelines for patient selection Day care surgery is possibly the clearest and most evident example of economy in any health care system ${ }^{[61,62]}$. Evidence supporting safety of major Day Care Surgery including laparoscopic cholecystectomy is predominantly composed of case series of selected patients ${ }^{[63,64]}$ Universal extrapolation of published experience is potentially dangerous because poor and unacceptable outcomes have been reported ${ }^{[66,67]}$ The risk may be even more in economically deprived countries where low literacy rates, lack of reliable and efficient transport, absence of organized referral patterns, poorly developed communication systems, underdeveloped primary health care services, Poor patient compliance and 
absence of community nursing. All these factors have prevented the successful introduction of major surgical operations as day care surgery. Even mortality has been

reported in ambulatory laparoscopic cholecystectomy. Unfortunately, these are the very

countries that could benefit most from day care surgery by reducing health care

expenditure and waiting times for elective surgery. Thereby sparing time and money to

be able to concentrate on other health related issues plaguing the particular society.

A major problem has been the absence of safe guidelines. There was little published experience of day care surgery from developing countries until recently. A

crucial aspect in the development of a safe Day Care program is the criteria for patient selection. Robinson's et $\mathrm{al}^{[44]}$ reported their experience in a public academic institution where they achieved outpatient laparoscopic cholecystectomy in $70 \%$ of unselected patients and they have identified ASA classification, procedural duration and surgery start time as a factor associated with failure of day care program. Some authors have come to the conclusion that appropriate patient selection lowers failure rate and patients most likely to fulfill the criteria of day care laparoscopic cholecystectomy are ASA grade I,II and younger patients.

Existing experience relates to practice in private health care centers where facilities are in accordance with published western guidelines ${ }^{[68,69]}$, A centre without previous experience, and without infrastructure to support major day care surgery, needs to evolve well reasoned guidelines with a rigid and honest appraisal of outcomes to identify deficiencies and potential pitfalls.

Table 8 summarises our results and compares them with the experience reportedin the literature.

Comparative results (table 8)

\begin{tabular}{|c|c|c|c|c|c|c|}
\hline Series & Total operated & $\begin{array}{l}\text { No } \\
\text { undergoing } \\
\text { DCLC }\end{array}$ & $\begin{array}{l}\text { Patients } \\
\text { eligible for } \\
\text { DCLC }(\%)\end{array}$ & $\begin{array}{l}\text { Successful } \\
\text { discharge } \\
(\%)\end{array}$ & $\begin{array}{l}\text { Readmission } \\
(\%)\end{array}$ & $\begin{array}{l}\text { Complications } \\
(\%)\end{array}$ \\
\hline \multicolumn{7}{|l|}{ *All minor. } \\
\hline Smith et $\mathrm{al}^{[39]}$ & 266 & 98 & 36 & 81 & - & $\overline{-}$ \\
\hline $\begin{array}{ll}\text { Prasad } & \text { and } \\
\text { Foley }^{[41]}\end{array}$ & 103 & 51 & 49 & 92 & - & $12 *$ \\
\hline Lillemoe $^{[64]}$ & NS & 130 & NS & 94 & 4.6 & - \\
\hline $\begin{array}{l}\text { Fiorillo et } \\
\mathrm{al}^{[70]}\end{array}$ & 238 & 149 & 63 & 61 & - & 2.1 \\
\hline $\begin{array}{l}\text { Stephenson et } \\
\mathrm{al}^{[65]}\end{array}$ & NS & 15 & NS & 80 & - & - \\
\hline $\begin{array}{l}\text { Mjaland et } \\
\text { al }^{[19]}\end{array}$ & NS & 200 & NS & 94 & 8 & 7 \\
\hline Present series & 50 & 45 & NA & 90 & 4 & $<1$ \\
\hline
\end{tabular}

The viability of our inclusion criteria and the feasibility of Day Care surgery in our practice was analysed using successful discharge, post operative symptoms readmission and complication rates, ease and accuracy of follow up and the potential benefit to our surgical service from Day Care surgery based on Day Care Laparoscopic Cholecystectomy as a model was examined.

Fifty patients met the criteria for eligibility. Of those found unsuitable, majority were rejected only because they had co morbid conditions and few patients simply refused to be discharged on the same day and the rest were overage. Patients coming from far off places were also excluded from the study This is common in developing countries and highlights a major problem in the organisation of day care surgery where there are no defined referral territories and where patients often have to travel to centers with better infrastructure, resources, and reputation. 45 out of 50 patients were successfully discharged thus superficially validating our recruitment criteria.

Though the onus of reaching the hospital for perioperative problems was on the patient, our results suggest a satisfactory outcome if patients and relatives understand the issues related to day care surgery and the response expected from them. It also appears that domiciliary perioperative care, even in our set-up, is feasible and reliable. Provided the pathways of care are clearly defined and understood, similar results can probably be achieved in most situations. In this context, our selection criteria appear to be both valid and practical.

Our study can be criticised on the grounds that patients were exposed to unwarranted risks by use of criteria that had not been validated. This criticism is valid and we concede that there should be better ways of undertaking feasibility studies. Unfortunately, there were no objective methods or models available to determine the safety of early discharge. There should be more strict and set pair of guidelines, especially where experience is small. It must be pointed out, however, that even minimally literate patients respond satisfactorily to the demands and responsibilities of day care surgery and should not be eliminated for this reason alone.

The main argument regarding day care laparoscopic cholecystectomy is the approach of some surgeons who prefer a period of at least 24 hours with overnight stay at hospital before discharge. They argue that it 
avoids delay in detection of complications in immediate postoperative period. On the contrary, other authors argue that the incidence of life threatening complication including arterial bleeding is very low 1:2000 and as such is symptomatic within hours during the post operative period thus being possibly detected while the patient is still in the hospital ${ }^{[71]}$. A complication such as biliary iatrogenic injury develops in laparoscopic cholecystectomy in $.3 \%-1 \%$ cases $^{[72]}$. If not detected intraoperatively, secondary symptoms develops including a biliary collection(abdominal pain, fever etc) or jaundice during the post operative period. These complications cannot be detected before the $2^{\text {nd }}$ post operative day. In our case no patient had an arterial bleed or a common bile duct injury or any related complications. This fact can be attributed to the exclusion of patients with radiological signs of acute cholecystitis from the study. No successfully discharged patient developed any major post operative complication. Therefore a period of prudent observation for 7-12 hours may suffice, due to the fact that overnight stay would not reduce the detection of subsequent major complications.

Cancellation on the day of surgery is an indirect indicator of the quality of day care services. Besides causing inconvenience to patients, it adversely affects surgical schedules thereby annulling the benefits of day care surgery. In addition, a high cancellation rate suggests deficient initial preanesthetic evaluation, which is potentially dangerous. Less than $1 \%$ of patients had their operation cancelled on the day of surgery. A few cancellations are acceptable because new clinical situations may arise after the earlier evaluation, especially if there is a significant time between evaluation and surgery; this happened in one of our patients. Since such changes in clinical status jeopardise patient safety, a close watch needs to be kept for co morbid conditions that are known to change (for example, hypertension) over short periods. Further, alteration of social conditions in the waiting period must also be looked into. These issues have not been adequately addressed in published literature; also, the optimal interval between preoperative anesthetic evaluation and surgery is uncertain. It has been recently suggested that this could be up to 30 days for low risk patients ${ }^{[73]}$. For high risk patients being considered for day care surgery, the evaluation probably needs to be done as close to the day of surgery as possible. There are no studies which have examined this issue and definite recommendations do not exist. While successful day care surgery has been reported in high risk patients ${ }^{[39,64]}$, the degree of selection exercised in these studies is uncertain. In most cases and certainly in conditions like ours, it is probably better to limit day care surgery to ASA grade I and II patients.

Unplanned admission after day care surgery is an indicator of quality assurance ${ }^{[74,75]}$ and patient selection; $4 \%$ of our patients needed readmission( 2 out of 50 ). Out of the two patients who required readmission one was complaining of severe nausea/vomiting and pain abdomen however the review ultrasound, routine blood tests and liver function tests were found to be normal. The other patient was admitted with the complain of post operative fever with pain abdomen her investigations were also found to be normal. Both the patients were managed conservatively and none required further surgical intervention. Similar experience has been reported,

in the literature ${ }^{[19]}$ and is not a specific problem of day care surgery. However it has been reported that the readmission rates can be lowered significantly by following strict norms while selecting the patients for day care. Post operative nausea and vomiting remained a frequent reason for unplanned admission after day care laparoscopic cholecystectomy. Hollington et al ${ }^{[76]}$ reported post operative nausea and vomiting as a frequent cause for unplanned admission after day care laparoscopic cholecystectomy. In our study 5 out of 50 patients could not be discharged from the hospital as day care cases. Two patients had severe nausea/vomiting, two had severe pain abdomen and one patient refused discharge due to individual preference The frequency of post operative pain, nausea and vomiting in our study was remarkably less, which can be attributed to proper patient selection and use of adequate preoperative and post operative analgesia, anxiolytics and prokinetic drugs.

Several authors recommend the use of standard protocols to minimize postoperative symptoms of pain, nausea or vomiting. Methods used to prevent nausea include avoiding the use of volatile anesthetic agents and undue use of opiates in the post operative period. Ramosetron was chosen as effective antiemetic in reducing postoperative nausea or vomiting ${ }^{[77,78]}$

Our criteria for discharge are therefore, satisfactory; the discharge parameters, based on published criteria, also appear to be reliable ${ }^{[4]}$. Follow up by telephone is the commonest and most cost effective method after day care surgery. If published guidelines were to be rigidly followed, countries with poor telecommunication infrastructure would necessarily have to restrict the ambit of day care surgery, negating its impact on health care delivery. Our data clearly show that follow up can be equally reliable if patients are asked to telephone the Hospital rather than the traditional method of medical teams contacting patients. During the study, all the patients who were part of our study were explained in detail about the complications and the due course of action, telephone number of the resident doctor was given to each patient and vice versa. All the patients were contacted by the doctor once they had reached home safely and thereafter as the need arised the patients inquired about their queries and problems from the resident doctor structured forms have been shown to be a better assessment tool compared with narrative notes and they also help in training medical personnel ${ }^{[79]}$. The most important benefit of day care surgery is reduction in health care expenditure but benefits 
are not necessarily similar for all countries. The cost of surgery in developing countries is low vis a vis those in developed countries. An expectation of reduction in costs would be rational but our data do not permit us to project a categorical benefit. There may, however, be another definite advantage of day care surgery that is relevant to surgical practice in developing countries as described ahead.

The number of surgical beds in any hospital, including ours, are relatively constant and non-flexible. Since our region has a high prevalence of gallstones, a large number of beds are used for cholecystectomy. This causes delays in the care of patients with more serious medical problems such as malignancies and emergencies. Additionally, many (up to 60\%) patients are from outside the proposed geographical area of our hospital. Repeated journeys to the hospital for evaluation and therapy increases cost of health care tremendously. Inpatient evaluation is often the most efficient approach to diagnosis and therapy in these groups but can often run into problems since beds may be blocked by patients with benign but widely prevalent surgical disease. Some prioritisation occurs covertly (as shown by the long waiting period for patients undergoing inadvernt cholecystectomy) to favor more serious illnesses but this may be unfair to patients with benign diseases like gallstones, because it delays treatment. Benign diseases often occur in those who are relatively young, who are in the most productive period of life, and who may have young dependents. Moreover, symptoms and complications of benign diseases including gallstones can be severe or even life threatening. It is therefore necessary to achieve an optimal balance without compromising care of groups with more complex disease. Our study has shown reduction in waiting times for patients undergoing Day Care surgery compared with inpatient cholecystectomy. The study has also transferred a large number of patients with gallstones (who would otherwise be treated as inpatients) to day care surgery thereby leading to better bed utilisation. This has also been the experience of other groups ${ }^{[61]}$. This outcome is theoretically beneficial but it is not a long term solution to the complex issue of equitable distribution of acceptable health care for the two groups and problems are bound to arise in the future. It is the recognition of this future problem that makes the results of our study so important. Our results should encourage additional studies to expand the list of procedures that can be brought under the umbrella of day care surgery. It should also provide health authorities with viable options while planning solutions and act as evidence based impetus for the development of exclusive day care facilities. In this way, the management of both simple and complex health problems could receive equitable attention and would become more efficient.

Comparision showing success, failure and readmission rates of various studies(table 9)

\begin{tabular}{|c|c|c|c|c|}
\hline Study & atients & Success rate(\%) & Failure rate $(\%)$ & Readmission rate $(\%)$ \\
\hline Selim et $\mathrm{al}^{[58]}$ & 200 & $90 \%$ & $10 \%$ & $10 \%$ \\
\hline Taylor et al[40] & 108 & $87 \%$ & $13 \%$ & na \\
\hline Robinson et al ${ }^{[44]}$ & 387 & $70 \%$ & $30 \%$ & na \\
\hline S.S. Bal et al ${ }^{[45]}$ & 313 & $92 \%$ & $8 \%$ & $3 \%$ \\
\hline Ibramin et $_{\text {al }}^{[46]}$ & 70 & $100 \%$ & $0 \%$ & $1.5 \%$ \\
\hline Chauhan et $\mathrm{al}^{[47]}$ & 287 & $96 \%$ & $4 \%$ & $3 \%$ \\
\hline Athar et al ${ }^{[54]}$ & 50 & $92 \%$ & $8 \%$ & $2 \%$ \\
\hline Kaman et al ${ }^{[60]}$ & 106 & $95 \%$ & $5 \%$ & na \\
\hline Present study & 50 & $90 \%$ & $10 \%$ & $4 \%$ \\
\hline
\end{tabular}

The results obtained by our study are comparable with the similar studies performed in India and abroad. Our results demonstrated that day care laparoscopic cholecystectomy is a safe and feasible procedure in public sector hospital in a developing country like India with high success rate and increased patient satisfaction in carefully selected patients with uncomplicated symptomatic cholelithiasis.

\section{Summary And Conclusion}

The present study was conducted at a public sector hospital in Punjab, India to evaluate the feasibility and outcome in 50 patients undergoing laparoscopic cholecystectomy as a Day Care surgery. The patients undergoing Day Care laparoscopic cholecystectomy were selected according to preset inclusion criteria. The patients, who satisfied the conditions for discharge, were discharged from the hospital on the very same day of surgery. Out of the 50 patients selected for Day care laparoscopic cholecystectomy, 45 patients were discharged from the hospital, on the same day of the surgery, 5 out of 50 patients could not be discharged from the hospital either due to nausea/vomiting, excessive pain abdomen or individual patient preference, 2 out of these 5 patients were readmitted to the hospital with the complain of nausea/vomiting with pain abdomen and post operative fever respectively. Thus we were able to achieve a success rate of $90 \%$ in our study which is comparable to all international standards. Younger and healthier patients seemed to be more suitable for the concept of Day Care surgery. The criteria of including ASA I, II and excluding ASA III, IV seemed to be valid from the conducted study. Though our criteria for selection and discharge seem to be valid, more strict guidelines and scales need to be devised for better patient selection and improved results. Early patient counseling and adequate risk explanation have led to a better patient acceptability. The importance of this need to be emphasized because in 
Day Care surgery the postoperative care of the patient shifts to the patients home. So it is utmost important that the patient should be explained in detail procedure related complications along with their signs and symptoms.

Day care surgery is a cost effective method, which not only reduces the hospital expenses but also decreases the patient morbidity with an early routine normal activity.

Day care surgery has also led to decrease in the wait list of patients for elective surgery with better hospital bed utilization. Thus providing better health care facilities. So more patients should be encouraged to join this program of Day Care surgery. More studies should be done in public sector hospitals across India to validate this concept and make it a regular feature of our health care system. As it will lead to better utilization of our health care resources. Through our study we found that Day Care laparoscopic cholecystectomy is a safe and feasible procedure in a public sector hospital in carefully pre-selected patients.

\section{References}

[1]. Keulmans Y, Eshius J, Haes H, Wit LT, Gouma DJ. Laparoscopic cholecystectomy: Day care versus clinical observation. Ann Surg 1998; 228:734-740.

[2]. Portincasa P, Moschetta A, Palasciano G. Cholesterol gallstone disease. Lancet 2006; $368: 230-239$.

[3]. Shehadi WH, The biliary system through the ages. Int Surg 1979; 64: 63-78

[4]. Beal JM. Historical perpective of gall stone disease. Surg Gynecol Obstet 1984; 158: 181.

[5]. Turner GG. The history of gall bladder surgery. Br Med J 1939; 1(4078): 464-465.

[6]. De U . evolution of cholecystectomy: A tribute to Carl August Langenbuch. Indian J Surg. 2004; 66 : $97-100$.

[7]. Karam J, Roslyn JR . Cholelithiasis and cholecystectomy. Maingot's abdominal operations. 12 th edn. Prentice Hall International Inc; 1997; 2: 1717-1738.

[8]. Polychronidis A, Laftsidis P, Bounovas A, Simopoulos C .Twenty years of laparoscopic cholecystectomy: Philippe Mouret March 17, 1987. JSLS 2008 Jan-Mar; 12(1): 109-111.

[9]. Morgenstern L, Lagenbuch C. The first cholecystectomy Surg Endosc Morgenstern L, Langenbuch C. The first cholecystectomy. Surg Endosc 1992;6:113-114

[10]. Lukihev OD, Filimonov MI, Zybin IM. A method of laparoscopic cholecystectomy. Khirurgia 1983; 8 : 125 - 127.

[11]. Muhe E. Die erste cholezystektomie durch das laparoscop. Langenb Arch Klin .1986; 369: 804.

[12]. Litynski GS. Erich muhe and rejection of laparoscopic cholecystectomy: A surgeon ahead of his time. JSLS 1998; 2: 341-46

[13]. Reynolds W. The first laparoscopic cholecystectomy. JSLS $2001 ;$ : 89-94

[14]. Meher G, Huttl TP. Laparoscopic surgery in Europe : Development and education. Surg Endosc 2001; 15: $229-31$.

[15]. Soper NJ, Stockman PT, Dunnegan DL, Ashley SW. Laparoscopic cholecystectomy: The new 'gold standard'. Arch Surg 1992; 127: 917-921.

[16]. White JV. Laparoscopic cholecystectomy The evolution of general surgery. Ann Intern med 1991; 115: $651-53$.

[17]. Maestroni U, Sortini D, Devito C, Pour Morad Kohan, Brunaldi F, Anania G, Pavanelli L, Pasqualucci A, Donini A. A new method of preemptive analgesia in laparoscopic cholecystectomy. Surg Endosc 2002; 16:1336-40.

[18]. Kum CK, Wong CW, Goh MY, Ti TK. Comparative study of pain level and analgesia requirements after laparoscopic and open

[19]. cholecystectomy. Surg Endosc 1994; 4:139-41.

[20]. Mjaland O, Reader J, Aesboe V, Trodsen E, Buanes T. Outpatient Laparoscopic cholecystectomy. Br J Surg 1997; 84:958-61.

[21]. Prof A. Darzi, Dept of health, U.K. The Day Care Surgery Operational Guide, August 2002;1-28.

[22]. Mueenullah K, Aliya A, Liala A, Azmeena N, Aslam F, Fauzia AK, unanticipated hospital admission after ambulatory surgery. Journal Pak Med Assoc 2005;55:251-52.

[23]. Zegarra RF II, Saba AK, Perchiera JL, Outpatient Laparoscopic cholecystectomy: safe and cost effective? Surg Laparosc Endosc

[24]. 1997; 7:487-90.

[25]. Skattum J, Edwin B, Tranden E, et al. Outpatient laparoscopic surgery: feasibility and consequences for education and health care

[26]. costs. Surg endosc 2004;18:796-801.

[27]. Friedman Z, Chung F, Wang DT. Ambulatory surgery adult patient selection criteria- a survey of Canadian anaesthesiologists . Can J Anaesth 2004;51:437-43.

[28]. Reddick EJ, Oslen DO, Outpatient laparoscopic laser cholecystectomy. American J Surg 1990; 160: 485-7.

[29]. Hung L, David B, Contemporary outcome of ambulatory laparoscopic cholecystectomy in a major teaching hospital. World J Surg

[30]. 2002;26:1117-21.

[31]. Gupta R , Bogra J, Kothari N, Kohli M, Postoperative analgesia with intraperitoneal fentanyl and bupivacaine: A randomized control trial. Canadian J Med 2010; 1(1):1-11.

[32]. Mirizzi PL. Operative cholangiography. Lancet $1938 ; 2: 366-69$

[33]. Saleh JW. Laparoscopy. Philadelphia. WB Saunders Co;1988: 7-8.

[34]. Thompson CJ, The history and evolution of surgical instruments. NY: Schuman's ; 1942. 89-93.

[35]. Gunning JE, Rosenzweig BA, Evolution of Endoscopic Surgery,In ; White RA, Klien SR, ediors. Endoscopic Surgery. Boston : Mosby YearBook, Inc; 1991, 1 - 9.

[36]. Berci G. History of Endoscopy.in: Berci G, editor, Endoscopy. NY: Appleton-Century-crofts; 1976:19-23.

[37]. Haubrich WS. History of endoscopy. In: Sivak MV, editor. Gastroenterologic Endoscopy. Philadelphia,PA:WB Saunders Co;

[38]. 1987:2-19.

[39]. Nadeau OE, Kampmeier OF. Endoscopy of abdomen: Abdominoscopy. A prelimnary study, including a summary of the literature and description of the technique. Syrg Gynecol Obstet 1925;41:259.

[40]. Philipi CJ, Fitzgibbons RJ, Salerno GM. Historical Review: Diagnostic laparoscopy to laparoscopic cholecystectomy and beyond. In:Zucker KA, editor. Surgical Laparoscopy. St Louis, MO: Quality Medical Publishing, Inc;1991:3-21.

[41]. Hasson HM. Open laparoscopy vs closed laparoscopy: A comparision of complication rates. Adv Planned Parenthood 1978; 13:4150 .

[42]. Lightdale CJ. Laparoscoy and biopsy in malingnant liver disease. Cancer 1982 (suppl 11):2672-75.

[43]. Warshaw AC, Tepper JE, Shipley WU. Laparoscopy in staging and Planning therapy for pancreatic cancer. Am J Surg 1986;151:76-80.

[44]. Smith R, Kolyn D, Pace R. Outpatient laparoscopic cholecystectomy. HPB Surg. 1994;7(4):261-264.

[45]. Taylor E, Gaw F, Kennedy C. Outpatient laparoscopic cholecystectomy feasibility. Laparosc Endosc Surg J 1996;6(2):73-77. 
[46]. 41. Prasad A, Foley RJ. Day case laparoscopic cholecystectomy: a safe and cost effective procedure. Eur J Surg.1996;162(1):4346.

[47]. Votik AJ. Is outpatient cholecystectomy safe for the higher risk elective patients?Surg Endosc 1997;11:1147-9.

[48]. 43. Dirkssen CD, Schmitz RF, Hans KM, Nieman FH, Hoogenboom LJ, Go PM. Ambulatory laparoscopic cholecystectomy is as effective as hospitalization and from a social prospective less expensive: a randomized study. Ned Tijdschr Geneeskd 2001Dec 15;145(50): $\quad 2434-9$.

[49]. Robinson TN, Biffl WL, Moore EE, Heimbach JK, Calkins CM, Burch JM.Predicting failure of outpatient laparoscopic cholecystectomy. Am J Surg 2002; 184:515-8.

[50]. Bal S, Reddy LG, Prashad R, Guleria R, Kashyap L. Feasibilty and safety of day care laparoscopic cholecystectomy in a developing [51]. country. Post Med J.2003;79(931):284-288.

[52]. Barut I, Tarhan OR, Cerci C, Bulbul M. Experience of ambulatory laparoscopic cholecystectomy in Turkish patients. Eur J Med 2005; 2(3):96-99.

[53]. Chauhan A, Mehrotra M, Bhatia PK, Baj B, Gupta AK. Day care laparoscopic cholecystectomy in a developing country. World J Surg. 2006;30(9):1690-1695; discussion 1696-1697.

[54]. Kasem A, Paix A, Grandy-Smith S, El-Hasani S. Laparoendosc Adv J Surg Tech A 2006 Aug;16(4):365-8.

[55]. 49. Sherigar JM, Irwin GW, Rathore MA, Khan A, Pillow K, Brown MG. Ambulatory laparoscopic cholecystectomy outcomes JSLS. 2006 Oct- Dec;10(4):473-478.

[56]. Berrevoet F, Biglari M, Sinove Y, De Baardemaeker L, Troisi, De Hemptinne B. Outpatient laparoscopic cholecystectomy in Belgium: what are we waiting for? Acta chir belg,2006,106,537-540.

[57]. Ibnouf M, Mahmoud M, Abdulgadir YA, Salama AA, ElTayabElAmri. Day case laparoscopic cholecystectomy in Sudan. Sudan JMS 2006; Vol 1, no 1.

[58]. Proske JM, Dagher I, Revitea C, Carloni A, Beauthier V, Labaille T, Vons C, Franco D. Day case laparoscopic cholecystectomy: results of 211 consecutive patients. Gastroenterol Clin Biol.2007 Apr;31(4):421-4.

[59]. Victorzon M, Tolonen P, Vuorialho T. Day case laparoscopic cholecystectomy: treatment of choice for selected patients? Surg Endosc 2007 Jan;21(1):70-3.

[60]. Athar Ali, Chawla T, Jamal A. Ambulatory laparoscopic cholecystectomy: Is it safe and cost effective? J Minim Access Surg 2009 Jan-Mar;5(1):8-13.

[61]. Briggs CD, Irving GB, Mann CD, Creswell A, Englert L, Peterson M, Cameron IC. Introduction of a Day case laparoscopic cholecystectomy service in the U.K.: A critical analysis of factors influencing same day discharge and contact with primary care providers. Ann R Cokk Surg Engl.2009 Oct;91(7):583-590.

[62]. Wu Ji, Kai Ding, Ling-Tang Li, Dan Wang, Ning Li, Jie-Shou Li. Outpatient versus inpatient laparoscopic cholecystectomy: a single center clinical analysis. Hepatobiliary Pancreat Dis Int 2010;9:60-64.

[63]. Singh DR, Joshi MR, Koirala U, Shrestha BR, Gautam B. Early experience of Day Care surgery in Nepal. J Nepal Med Assoc 2010;49(179):191-4.

[64]. Selim S, Congizhan SO. Day Case laparoscopic cholecystectomy: Is it safe and feasible procedure. Eur J Gen Med 2010;7(4):372376.

[65]. Athanasios M, Emmanouil S, Athanasia T, Nikolaos D, Georgios A, Georgios P, Theodosios T. Safety and effectiveness of outpatient laparoscopic cholecystectomy in a teaching hospital: a prospective study of 110 consecutive patients. BMC Research notes 2010;3:207.

[66]. Kamam L, Iqbal J, Bukhal I, Dahiya D, Singh R. Day care laparoscopic cholecystectomy: Next standard of treatment for Gall Stone Disease.Gastroenterology research 2011;4(6):257-261.

[67]. Boothe P, Finegan BA. Changing the admission process for elective surgery: an economic analysis. Can J Anaesth1998;42:391-4.

[68]. Fleisher LA, Yee K, Lillemoe KD, et al. Is outpatient laparoscopiccholecystectomy safe and cost effective? A model to study transition of care. Anesthesiology1999;90:1746-55.

[69]. Fiorillo MA, Davidson PG, Fiorillo JA, et al. 149 day care laparoscopic cholecystectomies. Surg Endosc1996;10:52:6.

[70]. Lillemoe KD. Laparoscopic cholecystectomy as a "true" outpatient procedure: initial experience in 130 consecutive patients. J Gastrointest Surg 1999;3:44-9.

[71]. Stephenson BM, Callander C, Sage M, et al. Feasibility of "day case" laparoscopic cholecystectomy. Ann R Coll Surg Engl1993;75:249-51.

[72]. Schloss MD, Tan AK, Schloss B, et al. Outpatient tonsillectomy and adenoidectomy: complications and recommendations. Int J Pediatr Otorhinolaryngol1994;30:115-22.

[73]. Mitchell RB, Pereira KD, Friedman NR. Outpatient tonsillectomy. Is it safe in children younger than age 13? Arch Otolaryngol Head Neck Surg1997;123:681-3.

[74]. Ramanujam TM, Uma G, Usha V, et al. Advantages and limitations of day surgery in children in a developing country. Pediatr Surg [75]. Int 1998;13:512-4.

[76]. Mandhan P, Shah A, Khan AW, et al. Outpatient pediatric surgery in a developing country. Journal of the Pakistan Medical

[77]. Association2000;50:220-4.

[78]. Fiorillo MA, Davidson PG, Fiorillo JA, et al. 149 day care laparoscopic cholecystectomies. Surg Endosc1996;10:52-6.

[79]. Deziel DJ, Millikan KW, Economou SG, Doolas A, Ko ST, Airan MC.Complications of laparoscopic Cholecystectomy: A national survey of 4292 hospitals and an analysis of 77604 cases. Am J Surg 1993;165:9-14.

[80]. Richardson M, Bell G, Fullarton G; West of Scotland Laparoscopic Cholecystectomy Audit Group. Incidence and nature of bile duct injuries following laparoscopic Cholecystectomy: An audit of 5913 cases. Br J Surg 1996;83:1356-60.

[81]. Pollard JB, Olson L. Early outpatient preoperative anaesthesia assessment: does it help to reduce operating room cancellations? Anesth Analg 1999;89:502-5.

[82]. Margovsky A. Unplanned admissions in day-case surgery as a clinical indicator for quality assurance. Aust N Z J Surg2000;70:21620.

[83]. Twersky R, Fishman D, Homel P. What happens after discharge?

[84]. Return hospital visits after DCS. Anesth Analg1997;84:319-24.

[85]. Hollington P, Toogood GJ, Padbury RT. A prospective randomized trial of day stay only versus overnight stay laparoscopic cholecystectomy. Aust N Z J Surg. 1999;69:841-3.

[86]. Raphael JH, Norton AC. Antiemetic efficacy of prophylactic ondansetron in laparoscopic surgery: A randomized double-blind comparison with metoclopramide. Br J Anesth. 1993;71:958-61.

[87]. Alexander R, Lovell AT, Seingry D, Jones RM. Comparison of ondansetron and droperidol in reducing postoperative nausea and vomiting associated with patient controlled analgesia. Anesthesia. 1995;50:1086-8. 
[88]. Kleinpell RM. Improving telephone follow-up after DCS. Journal of Perianaesthesia Nursing1997;12:336-40. 